\title{
Dosimetric characterization of a microDiamond detector in clinical scanned carbon ion beams
}

\author{
Marco Marinelli, G. Prestopino, ${ }^{\text {a) }}$ C. Verona, and G. Verona-Rinati \\ INFN_Dipartimento di Ingegneria Industriale, Università di Roma "Tor Vergata," Via del Politecnico 1, \\ Roma 00133, Italy \\ M. Ciocca, A. Mirandola, and A. Mairani \\ Fondazione CNAO, Strada Campeggi 53, Pavia 27100, Italy \\ L. Raffaele \\ INFN_Laboratori Nazionali del Sud, Via S. Sofia 62, Catania 95123, Italy and Fondazione CNAO, \\ Strada Campeggi 53, Pavia 27100, Italy \\ G. Magro \\ INFN_Dipartimento di Fisica, Università degli Studi di Pavia, Via U. Bassi 6, Pavia 27100, Italy \\ and Fondazione CNAO, Strada Campeggi 53, Pavia 27100, Italy
}

(Received 10 October 2014; revised 11 February 2015; accepted for publication 6 March 2015; published 31 March 2015)

Purpose: To investigate for the first time the dosimetric properties of a new commercial synthetic diamond detector (PTW microDiamond) in high-energy scanned clinical carbon ion beams generated by a synchrotron at the CNAO facility.

Methods: The detector response was evaluated in a water phantom with actively scanned carbon ion beams ranging from 115 to $380 \mathrm{MeV} / \mathrm{u}$ (30-250 mm Bragg peak depth in water). Homogeneous square fields of $3 \times 3$ and $6 \times 6 \mathrm{~cm}^{2}$ were used. Short- and medium-term ( 2 months) detector response stability, dependence on beam energy as well as ion type (carbon ions and protons), linearity with dose, and directional and dose-rate dependence were investigated. The depth dose curve of a $280 \mathrm{MeV} / \mathrm{u}$ carbon ion beam, scanned over a $3 \times 3 \mathrm{~cm}^{2}$ area, was measured with the microDiamond detector and compared to that measured using a PTW Advanced Markus ionization chamber, and also simulated using FLuKA Monte Carlo code. The detector response in two spread-out-Bragg-peaks (SOBPs), respectively, centered at 9 and $21 \mathrm{~cm}$ depths in water and calculated using the treatment planning system (TPS) used at CNAO, was measured.

Results: A negligible drift of detector sensitivity within the experimental session was seen, indicating that no detector preirradiation was needed. Short-term response reproducibility around 1\% (1 standard deviation) was found. Only $2 \%$ maximum variation of microDiamond sensitivity was observed among all the evaluated proton and carbon ion beam energies. The detector response showed a good linear behavior. Detector sensitivity was found to be dose-rate independent, with a variation below $1.3 \%$ in the evaluated dose-rate range. A very good agreement between measured and simulated Bragg curves with both microDiamond and Advanced Markus chamber was found, showing a negligible LET dependence of the tested detector. A depth dose curve was also measured by positioning the microDiamond with its main axis oriented orthogonally to the beam direction. A strong distortion in Bragg peak measurement was observed, confirming manufacturer recommendation on avoiding such configuration. Very good results were obtained for SOBP measurements, with a difference below $1 \%$ between measured and TPS-calculated doses. The stability of detector sensitivity in the observation period was within the experimental uncertainty.

Conclusions: Dosimetric characterization of a PTW microDiamond detector in high-energy scanned carbon ion beams was performed. The results of the present study showed that this detector is suitable for dosimetry of clinical carbon ion beams, with a negligible LET and dose-rate dependence. (C) 2015 American Association of Physicists in Medicine. [http://dx.doi.org/10.1118/1.4915544]

Key words: synthetic diamond detector, carbon ions, relative dosimetry

\section{INTRODUCTION}

Due to their unique physical and radiobiological properties, high-energy beams of charged nuclear particles offer superior advantages in achieving a higher conformal dose delivery in the planned target volume and the best sparing of surrounding healthy tissues, as compared with the highest technology conventional photon radiotherapy techniques. ${ }^{1-5}$

The number of dedicated proton and heavy-ion therapy facilities for the treatment of deep-seated and radio-resistant solid tumors is rapidly growing worldwide. ${ }^{6-8}$ 
While radiotherapy with protons can, nowadays, be considered as a well-established treatment modality, with a relatively high number of hospital-based centers in operation, ${ }^{8,9}$ heavier ion radiotherapy, mostly based on carbon ions, is gaining an increasing interest.

Heavy-ion radiation therapy using actively scanned carbon ion pencil beams, ${ }^{5,10}$ together with active energy modulation techniques by synchrotron machines, represents one of the most advanced cancer treatment modalities, although the knowledge of the relative biological effectiveness (RBE) for carbon ions is associated with uncertainties of the order of $20 \% .^{11}$ Indeed, eight centers are currently treating patients worldwide using carbon ion beams, and more than 13000 patients have been treated with this modality so far. However, even though carbon ions are known to produce favorable clinical outcomes in selected cases, such as head and neck radioresistant tumors, no clinical evidence to support the advantages of carbon ions over protons and photons is available yet, and randomized clinical trials are needed. ${ }^{12-14}$ Until then, carbon ion radiotherapy should be, therefore, considered as an experimental treatment modality.

To date, dosimetry of high-energy scanned carbon ions for quality assurance (QA) purposes and treatment planning verification still represents an open research issue, and it is associated with considerably larger uncertainties, as compared to dosimetry of low-LET and passive radiation delivery modalities. ${ }^{1,3,15,16}$ Current international dosimetry protocols available for high energy ion beam radiotherapy recommend absolute ion beam dosimetry to be based on ionization chamber measurements in water phantoms (IAEA TRS-398). ${ }^{17}$ For measurements under nonreference conditions, the use of other dosimeters is also considered, provided that the energy and LET dependence of the detector response are checked against ionization chambers. ${ }^{17} \mathrm{~A}$ comprehensive review on dosimetry of high energy charged particle beams can be found elsewhere. ${ }^{3,11}$ The main issue in detector selection in particle therapy is represented by the significant energy or LET dependence of most detectors, with the exception of the ionization chambers. The response from radiographic or radiochromic films, silicon diodes, and other available solid state detectors, such as thermoluminescence detectors (TLDs), exhibits strong energy or LET dependence, and their use is limited to conditions with nearly constant LET. ${ }^{11}$

In addition, scanned high energy charged particle beam dosimetry presents some specific challenges, particularly related to measurement efficiency. The use of a single-element dosimeter, requiring delivery of the whole $3 \mathrm{D}$ treatment field for each point, would be highly time-consuming, and multiple measurement points would be preferable. ${ }^{18}$ The pretreatment verification measurements of patient-specific plans by scanned high energy pencil ion beams are routinely performed by simultaneous measurements of the dose at a representative sample of points, by means of a limited number of pinpoint ionization chambers (24, typically) arranged in a 3D stack, ${ }^{19}$ calibrated in terms of dose to water. ${ }^{17}$ Such ionization chamber arrays are considered as a suitable instrument for 3D dosimetry in scanning ion beam radiotherapy, thanks to their energy independence and small detector size. However, this method has a scarce capability of field sampling (i.e., field coverage), due to chamber large spacing. On the other hand, for the existing 2D detector arrays based on a matrix of hundreds of ionization chambers, the relatively large size of each chamber (4-5 mm side) can cause a significant and undesired dose averaging effect in situations where very high dose gradients are generated, such as in intensity modulated particle therapy (IMPT) fields. Therefore, the development of innovative multielement 2D or even better $3 \mathrm{D}$ dosimetric systems, with very high spatial resolution and reduced LET and energy dependence, being able to simultaneously acquire dose measurements in a large number of points, is highly desirable to speed up the workflow and improve spatial accuracy of the measurements themselves.

Due to its outstanding properties, single crystal diamond has long been considered an ideal material for the construction of small volume high-resolution radiation detectors, for a wide range of beam qualities used in radiotherapy, including high energy particle beams. However, at the time of this work, no dosimetric data were available for synthetic single crystal diamond in heavy-ion beams. ${ }^{11}$ Spotty results were reported on a polycrystalline diamond based thermoluminescent detector ${ }^{20}$ and a natural diamond detector by PTW-Freiburg (type 60003, now discontinued). ${ }^{21}$

In the past years, the feasibility of prototype detectors based on a synthetic single crystal diamond diode (SCDD) was thoroughly investigated at Rome "Tor Vergata" University laboratories. The dosimetric properties of such device, now commercialized by PTW-Freiburg as microDiamond type 60019, were evaluated for clinical photon, electron, and high energy scattered proton radiation therapy beam dosimetry. ${ }^{22-28}$ In addition, a good detector-to-detector reproducibility was recently demonstrated by studying the response of five microDiamonds in small field photon irradiation conditions. ${ }^{29}$

In the present paper, the dosimetric properties of a PTW microDiamond in high energy scanned clinical ion beams have been evaluated at the Italian National Center for Oncological Hadron Therapy (CNAO). The reported characterization of a microDiamond point-like dosimeter is also intended as a preliminary feasibility study for forthcoming development of a multipixel two- or three-dimensional diamond-based dose verification system.

\section{MATERIALS AND METHODS}

\section{A. Irradiation setup}

Irradiations were performed at CNAO, the first hospitalbased hadrontherapy facility in Italy, using one of the available fixed horizontal beam lines. At CNAO, proton and carbon ion beams are accelerated by a synchrotron, and full 3-D pencil beam scanning, including active energy variation, is adopted as treatment delivery modality. Full technological and clinical details about the CNAO are reported elsewhere. ${ }^{18,30-32}$ In particular, for the absorbed dose to water determination under reference conditions and, consequently, the beam monitor calibration, the IAEA-TRS 398 recommendations ${ }^{17}$ were used, adapted following the formalism proposed by Jäkel et al. ${ }^{33}$ to take into account the specificities of particle pencil beam 
scanning. Reference conditions included the use of a Farmertype ionization chamber calibrated under Co-60 beams at a standard dosimetry laboratory (SSDL), monoenergetic beams, homogeneous $6 \times 6 \mathrm{~cm}^{2}$ scanned fields, and the measurement depth of $2 \mathrm{~cm}$ in water. The effective point of measurement of the ionization chamber ( $2.3 \mathrm{~mm}$ displacement, i.e., 0.75 times the chamber inner radius) was taken into account.

A set of five monoenergetic uniformly scanned carbon ion beams was evaluated: 115, 151, 209, 280, and $380 \mathrm{MeV} / \mathrm{u}$, corresponding to $30,50,90,150$, and $250 \mathrm{~mm}$ Bragg peak (BP) depths in water, respectively. Two proton beams, of 149 and $198 \mathrm{MeV}$ in energy (151 and $250 \mathrm{~mm}$ Bragg peak depths in water, respectively), were also used, in order to test the dependence of detector response on particle type. Pencil beam size in terms of full-width-at-half-maximum (FWHM) is energydependent, ranging from approximately 8 to $4 \mathrm{~mm}$ for carbon ions and from 22 to $7 \mathrm{~mm}$ for protons, in air at the isocentre. Square homogeneous monoenergetic scanned fields were used, $6 \times 6 \mathrm{~cm}^{2}$ and $3 \times 3 \mathrm{~cm}^{2}$ in size for carbon ions and $6 \times 6 \mathrm{~cm}^{2}$ for protons. The scanning step was set to 2 and $3 \mathrm{~mm}$ for carbon ions and protons, respectively. The number of particles per spot varied from $5 \times 10^{5}$ to $8 \times 10^{6}$ for carbon ions and $1 \times 10^{8}$ for protons. Maximum beam intensity was 5.3 $\times 10^{7}$ particles/s for carbon ions and $2.5 \times 10^{9}$ for proton beams. Two spread-out-Bragg-peaks (SOBPs) calculated using the syngo RT Planning (Siemens AG, Germany) treatment planning system (TPS), uniform in terms of biological dose, for a $6 \times 6 \times 6 \mathrm{~cm}^{3}$ cubic volume in a water phantom and centered at 9 and $21 \mathrm{~cm}$ depths, respectively, were also evaluated.

All tests were performed using a 3-D motorized water phantom (MP3-P, PTW-Freiburg, Germany), with a thin lateral entry window in PMMA. The water-equivalent thickness of the PMMA window was taken into account to define accurately the depth of the detector sensitive layer inside the water tank.

\section{B. Diamond dosimeter and electrometer}

A commercial PTW microDiamond type 60019 detector was used for the measurements reported in this work; this type of detector is based on the same device structure employed for the fabrication of previously reported SCDD dosimeter prototypes. ${ }^{22-24}$ The SCDD is designed to operate as a Schottky barrier photodiode, with no need of an external bias voltage applied (photovoltaic mode). Details on device technology, fabrication process, and detection mechanism can be found elsewhere. ${ }^{34}$ The detector sensitive volume is about $0.004 \mathrm{~mm}^{3}, 2.2 \mathrm{~mm}$ in diameter, and approximately $1 \mu \mathrm{m}$ thick. The detector reference measurement point was assumed to be at the center of the top SCDD plate surface, $1 \mathrm{~mm}$ below detector tip, according to manufacturer specifications. Taking into account the water-equivalent thickness of the entrance windows of both the water phantom $(5.8 \mathrm{~mm})$ and microDiamond $(1 \mathrm{~mm})$, the minimum depth achievable in water for the detector was $6.8 \mathrm{~mm}$; this depth was accurately achieved and set in the software controlling the movement of the detector, by pushing the detector itself against the inner wall of the phantom. The lasers installed in the treatment room were used to align the water tank, as well as the detector in the transversal plane with respect to the beam direction. All measurements were performed with the detector axis parallel to the beam axis (axial orientation), as recommended by the manufacturer for application in clinical photon and electron radiotherapy beams. In order to check the effectiveness of such recommendation in high energy particle dosimetry, the microDiamond was investigated with its main axis perpendicular to beam direction as well (radial orientation).

A PTW Unidos webline electrometer (secondary standard, reference class dosimeter), remotely controlled, was used for charge readings. In all cases, the microDiamond response was acquired once the irradiation field was completely painted.

\section{C. Measurement details}

A summary of the dosimetric measurements performed in the uniform scanning monoenergetic radiation fields evaluated in the present study is reported in Table I.

Short-term and medium-term detector response stability, dependence on beam energy and ion type (carbon ions and protons), linearity with dose, dose-rate dependence, and Bragg peak measurements were evaluated. Except for the latter, all measurements were performed with the measurement point of

TABLE I. Summary of the main dosimetric measurements performed with the microDiamond detector in uniformly scanned carbon ion monoenergetic beams. Measurements under proton beams were also performed, to check the dependence of the detector response on particle type.

\begin{tabular}{|c|c|c|c|c|c|c|c|c|c|c|}
\hline & & \multicolumn{6}{|c|}{${ }^{12} \mathrm{C}$ ions } & \multicolumn{3}{|c|}{ Protons } \\
\hline & \multicolumn{5}{|c|}{ Energy $(\mathrm{MeV} / \mathrm{u})$} & \multicolumn{2}{|c|}{$\begin{array}{l}\text { Field size } \\
\left(\mathrm{cm}^{2}\right)\end{array}$} & \multicolumn{2}{|c|}{$\begin{array}{l}\text { Energy } \\
(\mathrm{MeV})\end{array}$} & \multirow{2}{*}{$\begin{array}{l}\text { Field size } \\
\qquad \begin{array}{l}\left(\mathrm{cm}^{2}\right) \\
6 \times 6\end{array}\end{array}$} \\
\hline & 380 & 280 & 209 & 151 & 115 & $6 \times 6$ & $3 \times 3$ & 198 & 149 & \\
\hline Short-term reproducibility ${ }^{a}$ & & & & $\bullet$ & & $\bullet$ & & & & \\
\hline Medium-term stability ${ }^{a}$ & & $\bullet$ & & & & $\bullet$ & & & & \\
\hline $\begin{array}{l}\text { Energy and particle type } \\
\text { dependence }^{a}\end{array}$ & $\bullet$ & $\bullet$ & $\bullet$ & $\bullet$ & $\bullet$ & $\bullet$ & & $\bullet$ & $\bullet$ & $\bullet$ \\
\hline Linearity with dose $\mathrm{e}^{\mathrm{a}}$ & & $\bullet$ & & & & & $\bullet$ & & & \\
\hline Dose-rate dependence ${ }^{a}$ & & $\bullet$ & & & & & $\bullet$ & & & \\
\hline Bragg peak measurement & & $\bullet$ & & & & & $\bullet$ & & & \\
\hline
\end{tabular}

${ }^{a}$ The measurement point of the microDiamond detector was put at $20 \mathrm{~mm}$ depth in water. 
the microDiamond detector at the equivalent depth in water of $20 \mathrm{~mm}$, this representing the reference conditions for absorbed dose determination at CNAO. ${ }^{30}$ Two clinical SOBPs, centered at two different depths in water, were also evaluated.

The dependence on beam energy of the microDiamond detector response was evaluated in a $6 \times 6 \mathrm{~cm}^{2}$ painted carbon ion irradiation field $\left(2 \times 10^{6}\right.$ particles/spot delivered). The investigated energies were 115, 151, 209, 280, and $380 \mathrm{MeV} / \mathrm{u}$, and the corresponding delivered doses measured at the beginning of each experimental session using a calibrated PTW Farmer ionization chamber (model 30013) were 3.32, 1.9, 1.36, 1.09, and $0.9 \mathrm{~Gy}$, respectively. For each energy, five consecutive irradiations were performed, so that short-term reproducibility of the detector response was evaluated.

At the end of the irradiation sets with carbon ions, the beam line setting was switched to deliver protons. Square scanned irradiation fields of $6 \times 6 \mathrm{~cm}^{2}$ were used $\left(1 \times 10^{8}\right.$ protons/spot delivered in each case), corresponding to doses of 1.110 and 0.906 Gy for the two evaluated beam energies of 149 and $198 \mathrm{MeV}$, respectively.

Medium-term detector response stability was evaluated over a two-month period and five different experimental sessions, by repeating the measurement of five consecutive charge readings in the same irradiation condition, i.e., $280 \mathrm{MeV} / \mathrm{u}$ carbon ion beam $6 \times 6 \mathrm{~cm}^{2}$ in size square field. Two different sets of three consecutive irradiations (1.9 Gy each as delivered dose), respectively, performed at the beginning and the end of the first experimental session, using $151 \mathrm{MeV} / \mathrm{u}$ carbon ion beams, were also used as a warm-up test and provided a measure of the preirradiation dose potentially needed in order to achieve a stable response from the detector.

Linearity with dose, dose-rate dependence, and Bragg peak measurement were performed by irradiating a squared homogeneous field of $3 \times 3 \mathrm{~cm}^{2}$ with $280 \mathrm{MeV} / \mathrm{u}$ carbon ion beam. In this case, a smaller field size was used to spare beam time. Linearity with dose was investigated in a dose range from 0.27 to 8.72 Gy. Dose-rate dependence was evaluated in terms of linearity of microDiamond detector response as function of beam intensity (particles/s). The beam intensity was changed so that the maximum ion beam particle flux $\left(5.3 \times 10^{7}\right.$ particles/s, called I100) was reduced to about $1 / 2$ ( $2.65 \times 10^{7}$ particles $\left./ \mathrm{s}, \mathrm{I} 50\right)$ and $1 / 5\left(1.06 \times 10^{7}\right.$ particles $/ \mathrm{s}$,
I20). Three charge values were recorded in the above irradiation conditions, i.e., I20, I50, and I100 by delivering an overall dose of 1.09 Gy in each case.

The $\mathrm{BP}$ of a $3 \times 3 \mathrm{~cm}^{2} 280 \mathrm{MeV} / \mathrm{u}$ carbon ion beam was measured by means of the microDiamond detector in the standard axial orientation. The detector position was remotely changed in a way that measurement points were closer around the BP and in the distal fall-off, at the beginning of the nuclear fragmentation tail. At each depth, the whole irradiation field was painted by the scanned carbon ion beam. The corresponding dose delivered at the reference position of $20 \mathrm{~mm}$ in water was again 1.09 Gy. A PTW Advanced Markus plane parallel ionization chamber (type 34045), connected to the Unidos webline electrometer and operated at the bias voltage of $400 \mathrm{~V}$, was irradiated in the same conditions for comparison.

A depth dose curve was also measured in the same irradiation conditions with the microDiamond detector put in the radial orientation and compared to the one obtained in the axial orientation.

The microDiamond detector was finally used to measure the delivered dose at the center of the two SOBPs previously calculated using the TPS. The dose values obtained by the microDiamond detector using the average calibration factor resulting from the previous characterization were compared to those calculated.

\section{D. Monte Carlo (MC) simulations}

Depth dose distribution curves in a water phantom for the $3 \times 3 \mathrm{~cm}^{2}$ scanned $280 \mathrm{MeV} / \mathrm{u}$ carbon ion beam were also simulated using FLUKA MC code (2011.2b.6 version). ${ }^{35,36}$ The CNAO horizontal beam line, ${ }^{30}$ including the vacuum window and the beam monitoring system, has been modeled using the FLUKA combinatorial geometry following the design details. To allow the comparison of the calculated depth dose distribution curves with the experimental data, the sizes of the Advanced Markus chamber's collecting electrode (radius $=2.5 \mathrm{~mm}$ ) and the microDiamond (radius $=1.1 \mathrm{~mm}$ ) were taken into account in the simulations. Similarly to the method reported by Molinelli et al. ${ }^{18}$ in the adopted cylindrical scoring mesh inside a pure water tank, the radial scoring was restricted to the specific
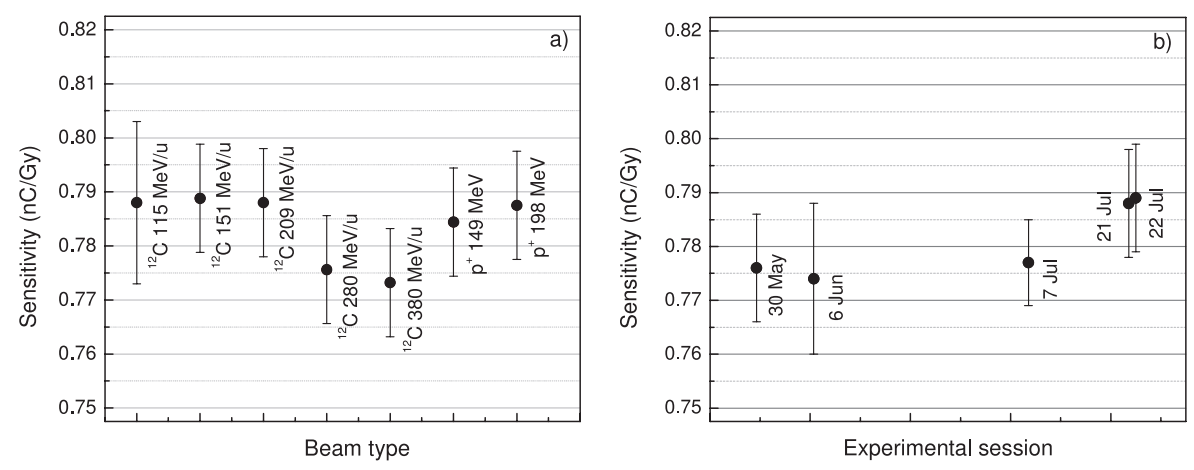

FIG. 1. (a) Variation of sensitivity values by microDiamond detector measured with the beam types evaluated in the present study. (b) Variation of detector sensitivity measured in a $6 \times 6 \mathrm{~cm}^{2} 280 \mathrm{MeV} / \mathrm{u}$ carbon ion beam, during five experimental sessions over a two-month period. In both cases, mean values (full circles) and error bars corresponding to the standard uncertainties $(k=1)$ are reported. 
active radius of the measuring device, hence deriving dose-towater in voxels of the same size as the detector volumes. The $\mathrm{MC}$ results were normalized at the depth in water of $20 \mathrm{~mm}$.

\section{RESULTS AND DISCUSSION}

\section{A. Response stability and energy dependence}

The warm-up test showed a small variation of $0.3 \%$, well within the experimental uncertainty, between the mean values of the detector response measured in the two irradiation sets (i.e., at the beginning and the end of the same experimental session, 5.7 Gy globally delivered in each case). This suggested that no preirradiation dose was needed to achieve a stable response for that specific detector, similarly to the finding reported by Mandapaka et al. ${ }^{24}$

The dependence of the detector response on both beam energy and particle type is shown in Fig. 1(a), where the mean values of the detector sensitivity and their standard uncertainties $(k=1)$ are reported for each set of charge readings. The overall relative standard deviation was around $1.3 \%$. Such a value is higher than the typical values (i.e., $0.5 \%$ ) obtained by using a reference Farmer ionization chamber in the same irradiation conditions. However, it should be pointed out that the dose locally delivered by the pencil beam in scanned ion beam facilities can be sensitive to even small fluctuations of the beam position along the scanning pattern. Such deviations are averaged out by a dosimeter whose dimension (approximately $23 \mathrm{~mm}$ in length and $6 \mathrm{~mm}$ in diameter for the Farmer chamber) is larger than the beam size, while they can be easily

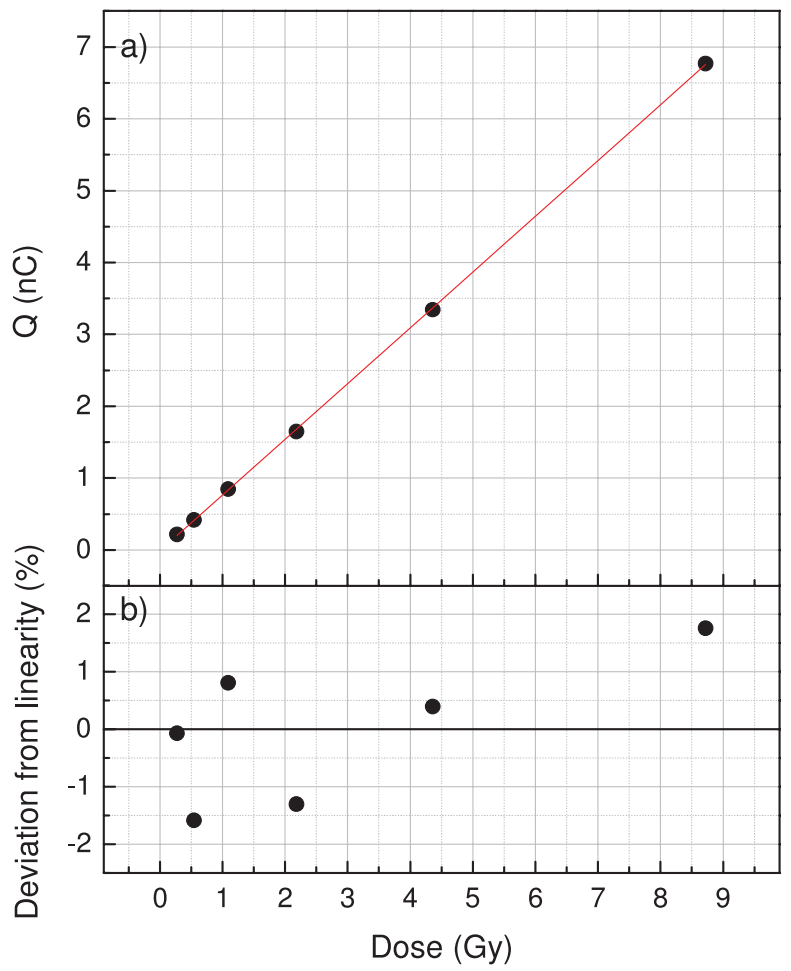

FIG. 2. (a) Charge measured by diamond detector and linear best fit (solid line) as function of the delivered dose in the $280 \mathrm{MeV} / \mathrm{u}{ }^{12} \mathrm{C}$ ion beam. (b) Percentage deviation from the linear best fit. appreciated by the microDiamond detector, whose active area is much smaller. As a consequence, the observed $1.3 \%$ overall standard deviation of the point-like doses measured by the microDiamond detector can be reasonably accounted for according to the above explanation. Indeed, much smaller standard deviation values were reported in the literature, as measured by premarket microDiamond prototypes under Co60 and scattered proton irradiations, ${ }^{24,37}$ where a repeatability of $0.1 \%-0.2 \%$ was reported, respectively. Among type B uncertainties, only the one due to both detector positioning at $20 \mathrm{~mm}$ depth in water and water-equivalent thickness of the detector entrance window, estimated as $0.3 \mathrm{~mm}$, was taken into account. Its relative contribution was only significant for the two lowest carbon ion beam energies $(1.4 \%$ and $0.3 \%$ at 115 and $151 \mathrm{MeV} / \mathrm{u}$, respectively). Although the determination of the microDiamond sensitivity was based on the detector calibration against the Farmer chamber under reference conditions for both carbon ion and proton beams, the relative standard uncertainty in the determination of the absorbed dose to water for cylindrical ionization chambers was not included in the error bars reported in Fig. 1. The IAEA estimated that uncertainty around $3 \%$ for carbon ion beams mainly dominated by those of water-to-air stopping power ratio and $W$-value, while $2 \%$ for protons. ${ }^{17}$ In other words, the authors made the basic assumption that the determination of the dose to water under reference conditions was performed correctly, while investigating the dosimetric properties of the tested detector.

The mean sensitivity of the microDiamond for carbon ion and proton beams was 0.783 and $0.785 \mathrm{nC} / \mathrm{Gy}$, respectively. Only $2 \%$ maximum variation was observed among all the evaluated carbon ion and proton beam energies. Therefore, the experimental data showed no appreciable dependence of the detector response on particle energy and type.

Figure 1(b) shows the medium-term stability of microDiamond response, measured over a two months period and in five different sessions, under the same irradiation conditions.

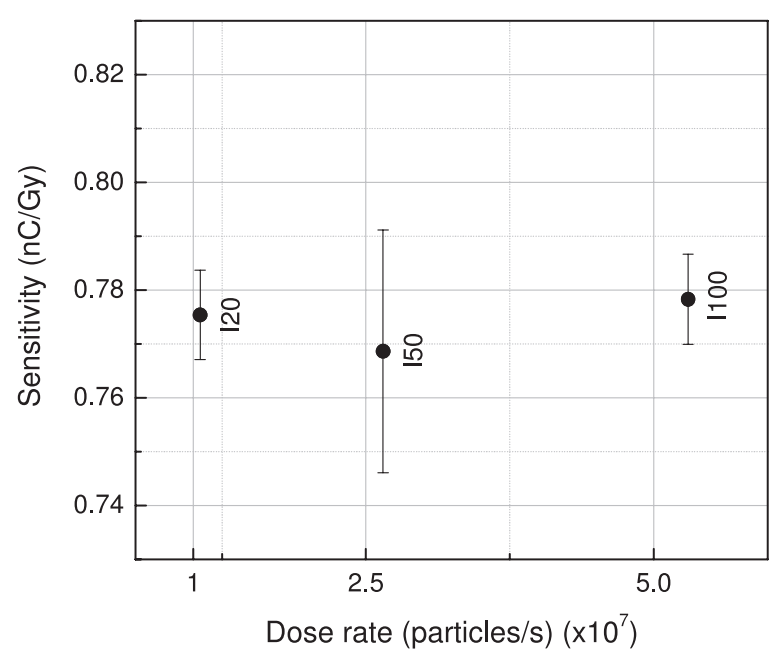

FIG. 3. Sensitivity of microDiamond detector as function of the beam dose rate. Mean values (full circles) of measured data are reported. Error bars correspond to the standard deviation of the experimental data for each evaluated dose rate. 
The standard deviations (1 ) and the mean values of each measured data set are shown. Maximum variation within $2 \%$ was found in the observation period, where a total dose of approximately $300 \mathrm{~Gy}$ was delivered to the detector. Although much longer beam time would be needed to exclude any radiation damage effect on the detector, these preliminary data seem to show a satisfactory response stability.

\section{B. Linearity with dose and dose-rate dependence}

The linearity with dose of the microDiamond detector response is shown in Fig. 2. Charge readings as a function of the delivered dose are reported in Fig. 2(a). A good linear behavior was observed, with the $R^{2}$ of the linear best fit equal to 1 with an accuracy of $10^{-5}$. A sensitivity of $(0.776$ $\pm 0.003) \mathrm{nC} / \mathrm{Gy}$ was calculated from the slope of the linear fit, which is consistent with the calibration factor calculated in the previously reported energy dependence measurements, in a different irradiation field. Deviation from linearity of the microDiamond response is shown in Fig. 2(b) as the

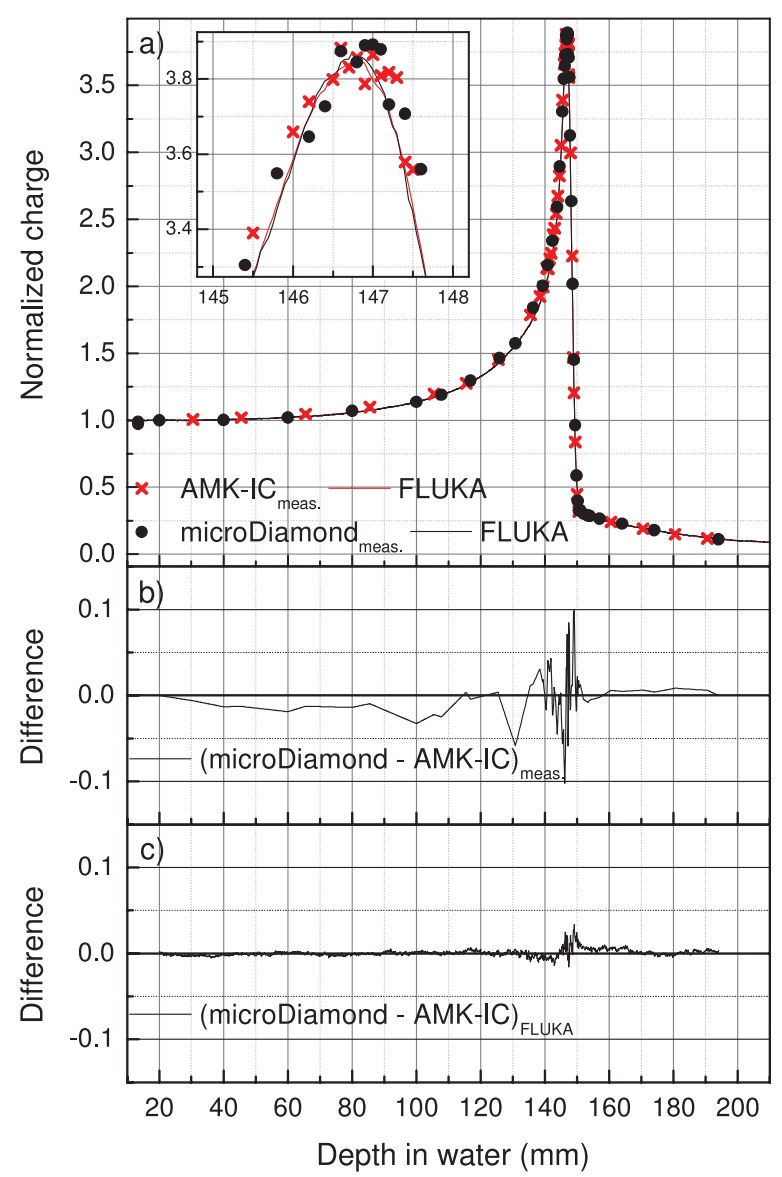

FIG. 4. (a) Bragg peak curves, normalized to 1 at the common depth in water of $20 \mathrm{~mm}$, of the $3 \times 3 \mathrm{~cm}^{2} 280 \mathrm{MeV} / \mathrm{u}$ carbon ion beam measured by microDiamond detector and PTW AMK-IC and calculated by FLUKA Monte Carlo code for both dosimeters. The sensitivity of the microDiamond at the depth of $20 \mathrm{~mm}$ was $0.771 \mathrm{nC} / \mathrm{Gy}$. (b) and (c) Difference plots between Bragg peaks measured by microDiamond and AMK-IC and calculated by FLUKA MC code, respectively. percentage deviation of the charge readings with respect to that resulting by the linear best fit. A variation less than $\pm 2 \%$ was found in the whole investigated dose range.

The dose-rate dependence of the microDiamond response is shown in Fig. 3. For each dose rate, i.e., for the three evaluated carbon ion beam particle fluxes (I100, I50, and I20), the error bars shown in Fig. 3 correspond to the variation (1) of each experimental data set with respect to the mean value. In the whole evaluated dose-rate range, the microDiamond sensitivity is shown to be constant within the experimental uncertainties. This indicates that no correction factors for doserate dependence are needed for the microDiamond in the investigated irradiation conditions.

\section{C. Bragg Peak measurement}

The BP curves of the $3 \times 3 \mathrm{~cm}^{2} 280 \mathrm{MeV} / \mathrm{u}$ scanned carbon ion beam are shown in Fig. 4, as measured by the microDiamond detector in axial orientation and by the reference PTW Advanced Markus ionization chamber (AMK-IC). Calculated Bragg peaks, obtained by simulating the irradiation setup and both tested detectors by means of FLUKA Monte Carlo code, are also shown in the same plot. A zoom of the BP curves in the vicinity of the Bragg peak is shown in the inset of Fig. 4(a). All measured and simulated curves are normalized to their respective plateau values, at $20 \mathrm{~mm}$ depth in water. A small adjustment of the depth in water was applied to the experimental curves (lower than $0.5 \mathrm{~mm}$ shift) to exactly match the depths of maximum dose among all the curves, thus compensating the uncertainty in detector positioning and its effective point of measurement. No detector response corrections were applied to the experimental microDiamond data, neither for doserate dependence nor for water-to-detector material stopping power ratios due to the small energy dependence of carbon to water stopping power ratios in the clinical energy range. ${ }^{38}$ Difference plots between the BP curves by microDiamond and

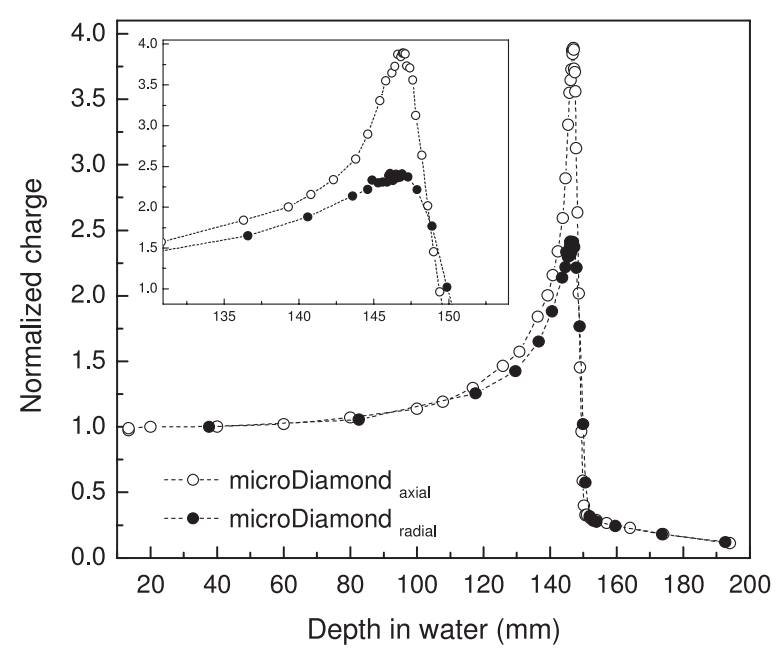

FIG. 5. Bragg peak curves, normalized at the entrance window, of the $3 \times 3 \mathrm{~cm}^{2} 280 \mathrm{MeV} / \mathrm{u}$ carbon ion beam measured by microDiamond detector in radial orientation. Measurement by microDiamond in axial orientation is also shown for comparison. 
TABLE II. Depth dose analysis parameters for the BPs shown in Figs. 4 and 5.

\begin{tabular}{lcccc}
\hline \hline & $\begin{array}{c}\text { Peak-to-Plateau } \\
\text { ratio }\end{array}$ & $\begin{array}{c}\text { Relative difference } \\
(\%)^{\mathrm{a}}\end{array}$ & $\begin{array}{c}\text { DDF } \\
(\mathrm{mm})^{\mathrm{b}}\end{array}$ & $\begin{array}{c}\text { BP width } \\
(\mathrm{mm})^{\mathrm{c}}\end{array}$ \\
\hline AMK-IC $_{\text {measurement }}$ & 3.85 & - & 1.67 & 2.88 \\
microDiamond $_{\text {measurement axial }}$ & 3.86 & 0.3 & 1.77 & 2.85 \\
AMK-IC $_{\text {FLUKA }}$ & 3.85 & - & 1.67 & 2.68 \\
microDiamond $_{\text {FLUKA }}$ & 3.86 & -37.7 & 1.66 & 2.63 \\
microDiamond $_{\text {measurement radial }}$ & 2.40 & 2.51 & 7.53 \\
\hline \hline
\end{tabular}

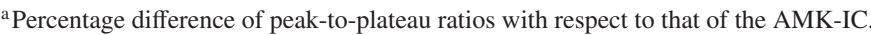

b $80 \%-20 \%$ distal-dose fall-off of the Bragg peak curves.

${ }^{\mathrm{c}}$ Referred to $80 \%-80 \%$ level.

AMK-IC are reported in Figs. 4(b) and 4(c), for the measured and simulated data, respectively. An overall very good agreement among BP curves, either measured or simulated, can be clearly observed.

The Bragg peak curve measured using the microDiamond detector in radial orientation is reported in Fig. 5. In this case, a $1.8 \mathrm{~mm}$ depth adjustment was applied to the curve. A strong distortion of the BP curve is observed in the case of the radial orientation. This can be ascribed to the much larger active volume dimension along the beam direction $(2.2 \mathrm{~mm}$ in radial orientation versus $1 \mu \mathrm{m}$ in the axial one). Indeed, a waterequivalent thickness of about $7 \mathrm{~mm}$ can be roughly estimated in this case, by taking into account the diamond/water stopping power ratios. This is believed to produce the volume averaging effect, resulting in the smearing and broadening of the Bragg peak observed in Fig. 5. The above result supports the manufacturer specifications, according to which the use of the microDiamond in radial orientation is not recommended. Even more so when high dose gradients are present along the beam axis direction, such as the ones observed in the Bragg peak region.

A quantitative analysis of all BP curves by microDiamond detector and AMK-IC shown in Figs. 4(a) and 5 is reported in Table II. In particular, the following features were derived: (i) the peak-to-plateau ratios of all the reported BP curves, (ii) the percentage differences of such ratios with respect to those obtained by AMK-IC, (iii) the distal-dose fall-off distances, defined as the distance of $80 \%$ and $20 \%$ distal points, and

TABLE III. Charge readings by microDiamond detector placed at the center of two uniform SOBPs $6 \times 6 \times 6 \mathrm{~cm}^{3}$ in volume, centered at 90 and $210 \mathrm{~mm}$ in water, respectively. Dose values were determined according to the mean calibration factor of detector response previously calculated. Measured data were compared to the doses calculated by the TPS.

\begin{tabular}{ccccc}
\hline \hline $\begin{array}{c}\text { SOBP depth } \\
(\mathrm{mm})\end{array}$ & $\begin{array}{c}\text { Charge } \\
(\mathrm{nC})\end{array}$ & $\begin{array}{c}\text { Dose micro- } \\
\text { Diamond } \\
(\mathrm{Gy})^{\mathrm{a}}\end{array}$ & $\begin{array}{c}\text { Dose TPS } \\
(\mathrm{Gy})\end{array}$ & $\begin{array}{c}\text { Relative } \\
\text { difference }(\%)^{\mathrm{b}}\end{array}$ \\
\hline 90 & 0.6775 & 0.865 & 0.868 & -0.3 \\
210 & 0.6745 & 0.861 & 0.869 & -0.9 \\
\hline \hline
\end{tabular}

${ }^{\mathrm{a}} 0.783 \mathrm{nC} / \mathrm{Gy}$ mean calibration factor for microDiamond detector response in carbon ion beams.

${ }^{\mathrm{b}}$ Percentage difference of estimated doses by microDiamond detector with respect to that by the TPS. (iv) the width of BP at $80 \%$ level. A relative difference of $0.3 \%$ was found in peak-to-plateau ratios calculated from both measured and simulated BP curves by microDiamond detector and AMK-IC, well below the experimental uncertainties. Such a small deviation confirmed the effectiveness of MC calculations and indicated that the microDiamond detector response is LET independent in the investigated energy range. This is consistent with previously reported results for a microDiamond prototype tested in high energy scattered proton beams. ${ }^{24}$ The observed LET independence may be ascribed to the very small thickness of the diamond diode depletion layer (about $1 \mu \mathrm{m}$ ), together with the relatively high electric field (built-in potential of about $1.3 \mathrm{~V}$ ). The combination of these two features is believed to drastically reduce the probability of carrier recombination and the space charge formation within the active volume. Such a behavior is markedly different from what reported in the literature for other solid state detectors proposed for dosimetry and quality assurance in high energy charged particle radiation therapy, such as films, ${ }^{39}$ TLDs, ${ }^{40}$ silicon diodes, ${ }^{41}$ alanine detectors, ${ }^{42}$ and diamonds, ${ }^{21}$ whose response is significantly affected by strong LET dependency.

Data derived from the Bragg peak curve measured by the microDiamond detector in radial orientation quantitatively confirm a strong underestimation of the peak-to-plateau ratio with respect to both the microDiamond in axial orientation and the AMK-IC, and a noticeable widening of the BP.

\section{D. SOBPs}

The results of the dosimetry test for the two $6 \times 6 \times 6 \mathrm{~cm}^{3}$ SOBPs are reported in Table III. Diamond response and its dose estimation were compared to the doses calculated by the TPS. The mean calibration factor of $0.783 \mathrm{nC} / \mathrm{Gy}$ for diamond detector response in the investigated carbon ion energy range was used for dose estimation (see Sec. 3.A). A maximum absolute difference of $0.9 \%$ with respect to the expected dose was observed, thus suggesting the suitability of microDiamond for carbon ion clinical treatment plan verification.

\section{SUMMARY AND CONCLUSIONS}

The dosimetric properties of a new commercial microDiamond detector were investigated in high-energy scanned clinical carbon ion beams for the first time. Detector response was 
studied in both uniform scanning monoenergetic carbon ion beams and in two different spread-out-Bragg-peaks delivered over a $6 \times 6 \times 6 \mathrm{~cm}^{3}$ cube volume. Proton beams were also used for the device characterization to test its dependence on particle type.

The microDiamond detector showed a stable response, with variations within the experimental uncertainties, as evaluated by both short-term and medium-term reproducibility measurements. A good behavior in terms of dependence on beam energy was found, with a maximum variation of the calibration factor lower than $2 \%$ over the evaluated carbon ion and proton beam energies investigated in the present work. A coefficient of determination $R^{2}$ equal to 1 with an accuracy of $10^{-5}$ was found when studying the device response as a function of the delivered dose. The detector sensitivity showed a maximum deviation from linearity within $\pm 2 \%$. The detector response was almost dose-rate independent, within the experimental uncertainties in the whole investigated dose-rate range. A very good agreement was found between depth dose curves measured by microDiamond and Advanced Markus ionization chamber, indicating a negligible LET dependence of the tested detector. A depth dose curve was measured with microDiamond in radial orientation as well, and a strong smearing and broadening of the measured Bragg peak were observed. Such result confirmed manufacturer recommendation on using the microDiamond in the axial orientation. Very good results were also found in SOBP dose measurements, with a relative difference below $1 \%$ between dose estimated by microDiamond measurement and the ones calculated by the TPS. It should be pointed out that detector-to-detector variability was not specifically investigated in the present work. However, the standardized microDiamond fabrication process and the reproducibility reported by Ralston et al. ${ }^{29}$ in the case of photon beams may suggest the validity of the above results for any microDiamond in hadrontherapy application.

In addition, more specific characterization would be required in order to assess the capability of the investigated device in terms of absolute dosimetry. Nonetheless, the obtained results indicate the microDiamond as a suitable detector for relative point dosimetry in modern high-energy actively scanned clinical carbon ion beams. Its negligible LET, dose rate, and energy dependence, together with its low preirradiation dose and high radiation hardness, represent very advantageous features as compared to the ones of other commercially available solid state detectors for ion beam dosimetry. Moreover, such features represent a promising basis for a forthcoming development of multipixel 2D dosimetric systems based on arrays of similar diamond Schottky diodes.

\section{ACKNOWLEDGMENTS}

This work is supported by the EMRP joint research project MetrExtRT which has received funding from the European Union on the basis of Decision No. 912/2009/EC. The EMRP is jointly funded by the EMRP participating countries within EURAMET and the European Union. The authors wish to thank PTW-Freiburg and Tema Sinergie for providing them the
microDiamond detector tested in the present study. This work was also jointly supported by "Fondazione Roma" through the INFN-DIARAD research project. Two of the authors, Marco Marinelli and Gianluca Verona Rinati, signed a contract with PTW-Freiburg involving financial interests deriving from the microDiamond dosimeter commercialization.

a) Author to whom correspondence should be addressed. Electronic mail: giuseppe.prestopino@uniroma2.it

${ }^{1}$ D. Schardt, T. Elsässer, and D. Schulz-Ertner, "Heavy-ion tumor therapy: Physical and radiobiological benefits," Rev. Mod. Phys. 82, 383-425 (2010).

${ }^{2}$ S. Braccini, "Scientific and technological development of hadrontherapy," e-print arXiv:1001.0860 (2010)

${ }^{3}$ C. M. Ma and T. Lomax, Proton and Carbon Ion Therapy (CRC, Boca Raton, FL, 2013).

${ }^{4}$ U. Amaldi, R. Bonomi, S. Braccini, M. Crescenti, A. Degiovanni, M. Garlasche, A. Garonna, G. Magrin, C. Mellace, P. Pearce, G. Pittà, P. Puggioni, E. Rosso, S. Verdú Andrés, R. Wegner, M. Weiss, and R. Zennaro, "Accelerators for hadrontherapy: From Lawrence cyclotrons to linacs," Nucl. Instrum. Methods Phys. Res., Sect. A 620, 563-577 (2010).

${ }^{5}$ M. Durante and J. S. Loeffler, "Charged particles in radiation oncology," Nat. Rev. Clin. Oncol. 7, 37-43 (2010).

${ }^{6}$ See http://www.ptcog.ch/index.php/facilities-in-operation.

${ }^{7}$ H. M. Kooy and T. F. Delaney, Proton and Charged Particle Radiotherapy (Lippincott Williams and Wilkins, Philadelphia, PA, 2007).

${ }^{8}$ U. Amaldi and G. Kraft, "Radiotherapy with beams of carbon ions," Rep. Prog. Phys. 68, 1861-1882 (2005).

${ }^{9}$ M. Goitein, A. Lomax, and E. Pedroni, “Treating cancer with protons," Phys. Today 55(9), 45-50 (2002).

${ }^{10}$ T. Haberer, W. Becher, D. Schardt, and G. Kraft, "Magnetic scanning system for heavy ion therapy," Nucl. Instrum. Methods Phys. Res., Sect. A 330, 296-305 (1993).

${ }^{11}$ C. P. Karger, O. Jäkel, H. Palmans, and T. Kanai, "Dosimetry for ion beam radiotherapy," Phys. Med. Biol. 55, R193-R234 (2010).

${ }^{12}$ T. Kamada, H. Tsujii, E. A. Blakely, J. Debus, W. De Neve, M. Durante, O. Jäkel, R. Mayer, R. Orecchia, R. Pötter, S. Vatnitsky, and W. T. Chu, "Carbon ion radiotherapy in japan: An assessment of 20 years of clinical experience," Lancet Oncol. 16, e93-e100 (2015).

${ }^{13}$ D. Schulz-Ertner, C. P. Karger, A. Feuerhake, A. Nikoghosyan, S. E. Combs, O. Jäkel, L. Edler, M. Scholz, and J. Debus, "Effectiveness of carbon ion radiotherapy in the treatment of skull-base chordomas," Int. J. Radiat. Oncol., Biol., Phys. 68, 449-457 (2007).

${ }^{14}$ D. Schulz-Ertner, A. Nikoghosyan, H. Hof, B. Didinger, S. E. Combs, O. Jäkel, C. P. Karger, L. Edler, and J. Debus, "Carbon ion radiotherapy of skull base chondrosarcomas,” Int. J. Radiat. Oncol., Biol., Phys. 67, 171-177 (2007).

${ }^{15}$ C. P. Karger, O. Jäkel, P. Heeg, and G. H. Hartmann, "Clinical dosimetry for heavy ion therapy," Z. Med. Phys. 12, 159-169 (2002).

${ }^{16}$ O. Jäkel, G. H. Hartmann, C. P. Karger, P. Heeg, and J. Rassow, "Quality assurance for a treatment planning system in scanned ion beam therapy," Med. Phys. 27, 1588-1600 (2000).

${ }^{17}$ IAEA (International Atomic Energy Agency), "Absorbed dose determination in external beam radiotherapy: An international code of practice for dosimetry based on standards of absorbed dose to water," Technical Report Series No. 398 (IAEA, Vienna, 2000).

${ }^{18}$ S. Molinelli, A. Mairani, A. Mirandola, G. Vilches Freixas, T. Tessonnier, S. Giordanengo, K. Parodi, M. Ciocca, and R. Orecchia, "Dosimetric accuracy assessment of a treatment plan verification system for scanned proton beam radiotherapy: One-year experimental results and Monte Carlo analysis of the involved uncertainties," Phys. Med. Biol. 58, 3837-3847 (2013).

${ }^{19}$ C. P. Karger, O. Jäkel, and G. H. Hartmann, "A system for three-dimensional dosimetric verification of treatment plans in intensity-modulated radiotherapy with heavy ions," Med. Phys. 26, 2125-2132 (1999).

${ }^{20}$ M. Rębisz, B. Voss, A. Heinz, E. Usenko, and M. Pomorski, "CVD diamond dosimeters for heavy ion beams," Diamond Relat. Mater. 16, 1070-1073 (2007).

${ }^{21}$ M. Sakama, T. Kanai, Y. Kase, M. Komori, A. Fukumura, and T. Kohno, "Responses of a diamond detector to high-LET charged particles," Phys. Med. Biol. 50, 2275-2289 (2005). 
${ }^{22}$ I. Ciancaglioni, Marco Marinelli, E. Milani, G. Prestopino, C. Verona, G. Verona-Rinati, R. Consorti, A. Petrucci, and F. De Notaristefani, "Dosimetric characterization of a synthetic single crystal diamond detector in clinical radiation therapy small photon beams," Med. Phys. 39, 4493-4502 (2012).

${ }^{23}$ C. Di Venanzio, Marco Marinelli, E. Milani, G. Prestopino, C. Verona, G. Verona-Rinati, M. D. Falco, P. Bagalà, R. Santoni, and M. Pimpinella, "Characterization of a synthetic single crystal diamond schottky diode for radiotherapy electron beam dosimetry," Med. Phys. 40, 021712 (9pp.) (2013).

${ }^{24}$ A. K. Mandapaka, A. Ghebremedhin, B. Patyal, Marco Marinelli, G. Prestopino, C. Verona, and G. Verona-Rinati, "Evaluation of the dosimetric properties of a synthetic single crystal diamond detector in high energy clinical proton beams," Med. Phys. 40, 121702 (9pp.) (2013).

${ }^{25}$ G. Azangwe, P. Grochowska, D. Georg, J. Izewska, J. Hopfgartner, W. Lechner, C. E. Andersen, A. R. Beierholm, J. Helt-Hansen, H. Mizuno, A. Fukumura, K. Yajima, C. Gouldstone, P. Sharpe, A. Meghzifene, and H. Palmans, "Detector to detector corrections: A comprehensive experimental study of detector specific correction factors for beam output measurements for small radiotherapy beams," Med. Phys. 41, 072103 (16pp.) (2014).

${ }^{26}$ J. E. Morales, S. B. Crowe, R. Hill, N. Freeman, and J. V. Trapp, "Dosimetry of cone-defined stereotactic radiosurgery fields with a commercial synthetic diamond detector," Med. Phys. 41, 111702 (6pp.) (2014).

${ }^{27}$ P. Papaconstadopoulos, F. Tessier, and J. Seuntjens, "On the correction, perturbation and modification of small field detectors in relative dosimetry," Phys. Med. Biol. 59, 5937-5952 (2014).

${ }^{28}$ J. M. Lárraga-Gutiérrez, P. Ballesteros-Zebadúa, M. Rodríguez-Ponce, O. A. García-Garduño, and O. O. Galván de la Cruz, "Properties of a commercial PTW-60019 synthetic diamond detector for the dosimetry of small radiotherapy beams," Phys. Med. Biol. 60, 905-924 (2015).

${ }^{29}$ A. Ralston, M. Tyler, P. Liu, D. Mckenzie, and N. Suchowerska, "Overresponse of synthetic microDiamond detectors in small radiation fields," Phys. Med. Biol. 59, 5873-5881 (2014).

${ }^{30}$ S. Rossi, “The status of CNAO,” Eur. Phys. J. Plus 126, 78 (2011).

${ }^{31}$ S. Giordanengo, M. Donetti, F. Marchetto, A. Ansarinejad, A. Attili, F. Bourhaleb, F. Burini, R. Cirio, P. Fabbricatore, F. Voelker, M. A. Garella, M. Incurvati, V. Monaco, J. Pardo, C. Peroni, G. Russo, R. Sacchi, G. Taddia, and A. Zampieri, "Performances of the scanning system for the CNAO center of oncological hadron therapy," Nucl. Instrum. Methods Phys. Res., Sect. A 613, 317-322 (2010).
${ }^{32}$ S. Giordanengo, M. Donetti, M. A. Garella, F. Marchetto, G. Alampi, A. Ansarinejad, V. Monaco, M. Mucchi, I. A. Pecka, C. Peroni, R. Sacchi, M. Scalise, C. Tomba, and R. Cirio, "Design and characterization of the beam monitor detectors of the italian national center of oncological Hadrontherapy (CNAO)," Nucl. Instrum. Methods Phys. Res., Sect. A 698, 202-207 (2013).

${ }^{33}$ O. Jäkel, G. H. Hartmann, C. P. Karger, P. Heeg, and S. Vatnitsky, "A calibration procedure for beam monitors in a scanned beam of heavy charged particles," Med. Phys. 31, 1009-1013 (2004).

${ }^{34}$ S. Almaviva, Marco Marinelli, E. Milani, G. Prestopino, A. Tucciarone, C. Verona, G. Verona-Rinati, M. Angelone, M. Pillon, I. Dolbnya, K. Sawhney, and N. Tartoni, "Chemical vapor deposition diamond based multilayered radiation detector: Physical analysis of detection properties,” J. Appl. Phys. 107, 014511 (2010).

${ }^{35}$ A. Ferrari, P. R. Sala, A. Fassò, and J. Ranft, "Fluka: A multi-particle transport code," CERN Yellow Report CERN 2005-10 (CERN, Geneva, 2005).

${ }^{36}$ G. Battistoni, F. Cerutti, A. Fassò, A. Ferrari, S. Muraro, J. Ranft, S. Roesler, and P. R. Sala, "The FLUKA code: Description and benchmarking," AIP Conf. Proc. 896, 31-49 (2007).

${ }^{37}$ M. Pimpinella, I. Ciancaglioni, R. Consorti, C. Di Venanzio, A. S. Guerra, A. Petrucci, A. Stravato, and G. Verona-Rinati, "A synthetic diamond detector as transfer dosimeter for $D_{w}$ measurements in photon beams with small field sizes," Metrologia 49, S207-S210 (2012).

${ }^{38}$ A. Lühr, D. C. Hansen, N. Sobolevsky, H. Palmans, S. Rossomme, and N. Bassler, "Fluence correction factors and stopping power ratios for clinical ion beams," Acta Oncol. 50, 797-805 (2011).

${ }^{39} \mathrm{M}$. Martišíková and O. Jäkel, "Dosimetric properties of gafchromic EBT films in monoenergetic medical ion beams," Phys. Med. Biol. 55, 3741-3751 (2010).

${ }^{40}$ O. B. Geiß, M. Krämer, and G. Kraft, "Efficiency of thermoluminescent detectors to heavy charged particles," Nucl. Instrum. Methods Phys. Res., Sect. B 142, 592-598 (1998).

${ }^{41}$ F. J. Kaiser, N. Bassler, and O. Jäkel, "COTS silicon diodes as radiation detectors in proton and heavy charged particle radiotherapy," Radiat. Environ. Biophys. 49, 365-371 (2010).

${ }^{42}$ R. Herrmann, O. Jäkel, H. Palmans, P. Sharpe, and N. Bassler, "Dose response of alanine detectors irradiated with carbon ion beams," Med. Phys. 38, 1859-1866 (2011). 